\title{
Normalization of Blood Pressure With Spinal Cord Epidural Stimulation After Severe Spinal Cord Injury
}

\author{
Susan J. Harkema ${ }^{1,2 *}$, Siqi Wang ${ }^{2}$, Claudia A. Angeli,1,2, Yangsheng Chen ${ }^{2}$, \\ Maxwell Boakye ${ }^{3}$, Beatrice Ugiliweneza ${ }^{3}$ and Glenn A. Hirsch ${ }^{4}$
}

${ }^{1}$ Frazier Rehab Institute, Louisville, KY, United States, ${ }^{2}$ Department of Neurological Surgery, Kentucky Spinal Cord Injury Research Center, University of Louisville, Louisville, KY, United States, ${ }^{3}$ Department of Neurosurgery, School of Medicine, University of Louisville, Louisville, KY, United States, ${ }^{4}$ Division of Cardiology, Department of Medicine, University of Louisville School of Medicine, Louisville, KY, United States

Chronic low blood pressure and orthostatic hypotension remain challenging clinical issues after severe spinal cord injury (SCl), affecting health, rehabilitation, and quality of life. We previously reported that targeted lumbosacral spinal cord epidural stimulation (scES) could promote stand and step functions and restore voluntary movement in patients with chronic motor complete SCI. This study addresses the effects of targeted scES for cardiovascular function (CV-scES) in individuals with severe SCl who suffer from chronic hypotension. We tested the hypothesis that CV-scES can increase resting blood pressure and attenuate chronic hypotension in individuals with chronic cervical SCl. Four research participants with chronic cervical SCl received an implant of a 16-electrode array on the dura (L1-S1 cord segments, T11-L1 vertebrae). Individual-specific CV-scES configurations (anode and cathode electrode selection, voltage, frequency, and pulse width) were identified to maintain systolic blood pressure within targeted normative ranges without skeletal muscle activity of the lower extremities as assessed by electromyography. These individuals completed five 2-h sessions using CV-scES in an upright, seated position during measurement of blood pressure and heart rate. Noninvasive continuous blood pressure was measured from a finger cuff by plethysmograph technique. For each research participant there were statistically significant increases in mean arterial pressure in response to CV-scES that was maintained within normative ranges. This result was reproducible over the five sessions with concomitant decreases or no changes in heart rate using individual-specific CV-scES that was modulated with modest amplitude changes throughout the session. Our study shows that stimulating dorsal lumbosacral spinal cord can effectively and safely activate mechanisms to elevate blood pressures to normal ranges from a chronic hypotensive state in humans with severe SCI with individual-specific CV-scES.

Keywords: spinal cord injuries, epidural stimulation, cardiovascular system, systolic blood pressure, heart rate

\footnotetext{
Abbreviations: AIS, American spinal cord injury association impairment scale; CV-scES, spinal cord epidural stimulation targeted specifically for cardiovascular function; post-CV-scES, time period following epidural stimulation targeted specifically for cardiovascular function; pre-CV-scES, time period prior to beginning epidural stimulation targeted specifically for cardiovascular function; scES, spinal cord epidural stimulation; SCI, spinal cord injury; stand-scES, standing targeted spinal cord epidural stimulation; Vol-scES, voluntary movement targeted spinal cord epidural stimulation.
} 


\section{INTRODUCTION}

Cardiovascular dysfunction is a leading cause of death in individuals with spinal cord injury (SCI) and has a significant negative impact throughout their lifetime. Individuals with chronic SCI above T6 experience persistent hypotension and bradycardia, with orthostatic hypotension and severe increases in blood pressure during autonomic dysreflexia resulting in drastic daily fluctuations in cardiovascular activity (Wecht and Bauman, 2017). This dysregulation of the autonomic system affects their quality of life by causing discomfort, disrupting their ability to participate in rehabilitation, and interfering with their engagement in daily activities of life. Symptoms of chronic low blood pressure and orthostatic hypotension include fatigue, light-headedness, dizziness, blurred vision, dyspnea, and restlessness associated with cerebral hypo-perfusion (Krassioukov and Claydon, 2006).

The management of chronic hypotension in SCI is a challenging clinical issue as there is no standard or fully successful management strategy. Pharmacological interventions use agents that increase the sympathetic stimulation of the cardiovascular system or increase the blood volume in circulation (Freeman, 2008; Krassioukov et al., 2009). Undesirable side effects of pharmacological interventions and requirement of planning ahead of orthostatic stress have limited their application. Some non-pharmacological interventions have been used with limited success, which include applying external compression to reduce venous pooling in the abdomen or the lower extremities with the elastic compression stocking and abdominal binders (Mathias and Kimber, 1998). Other interventions to improve orthostatic hypotension include functional electrical stimulation (Chao and Cheing, 2005), stand locomotor training (Harkema et al., 2008), and respiratory motor training (Aslan et al., 2016). Although these interventions obtained different degrees of success in reducing the severity of cardiovascular dysfunction, none have fully restored chronic hypotension.

In studies of motor behavior in individuals with motor complete SCI, we observed modulation of cardiovascular parameters when using scES at spinal segments L1-S1 (vertebrae T11-L1) that allows a direct modulation of the spinal neural networks below the level of injury. Targeted scES optimized for standing (stand-scES) enabled four individuals with chronic motor complete SCI to achieve full weight-bearing standing with minimal assistance (Harkema et al., 2011; Rejc et al., 2015, 2017). Targeted scES optimized for voluntary movement (Vol-scES) enabled the same four individuals to perform intentional joint movements (Harkema et al., 2011; Angeli et al., 2014).
These studies not only demonstrated effects of scES on altering the excitability of the spinal neural networks to process sensory and supraspinal inputs to improve motor behavior, but also highlighted the importance of optimization of the stimulation configurations, i.e., electrode set, frequency, and intensity, to activate the neuronal pools to achieve targeted tasks and the stimulation parameters were also specific to individuals. During these studies we observed in two individuals who had orthostatic hypotension, blood pressure was elevated and symptoms of orthostatic hypotension were prevented during standing and stepping (unpublished observations).

There have been animal studies (Sato et al., 2002; Yanagiya et al., 2004) and a proof of principle human study in neutrally intact individuals (Yamasaki et al., 2006) showing that scES of the lumbar spinal cord has the potential to ameliorate chronic hypotension. In this study we proposed to use targeted scES optimized for solely cardiovascular function (CV-scES) to provide a unique way to address cardiovascular dysfunction experienced in individuals with SCI. We hypothesized that CV-scES at spinal cord segments L1-S1 with specific targeted configurations can increase resting blood pressure and attenuate orthostatic hypotension in individuals with chronic cervical SCI.

\section{MATERIALS AND METHODS}

\section{Research Participants}

Four research participants with chronic motor complete, cervical SCI were studied (Table 1). All individuals were clinically stable, presented with orthostatic hypotension, persistent low resting blood pressure, and routine symptoms of autonomic dysreflexia with no cardiovascular disease unrelated to SCI. The research participants signed an informed consent prior to participating in the study. The study protocol and informed consent were approved by the University of Louisville Institutional Review Board in accordance with the Declaration of Helsinki. A 16-electrode array (5-6-5 Specify, Medtronic, Minneapolis, $\mathrm{MN}$, United States) was implanted to span the spinal cord segments L1-S1 (vertebrae T11-L1) in all research participants (Figure 1), as previously described (Harkema et al., 2011; Angeli et al., 2014). The electrode lead was tunneled subcutaneously and connected to the pulse generator (RestoreADVANCED, Medtronic, Minneapolis, MN, United States) placed ventral in the abdomen.

Research participants were resting in a seated position for $2 \mathrm{~h}$ with continuous blood pressure and heart rate monitoring. CV-scES configurations (anode and cathode electrode selection,

TABLE 1 | Characteristics of SCI participants.

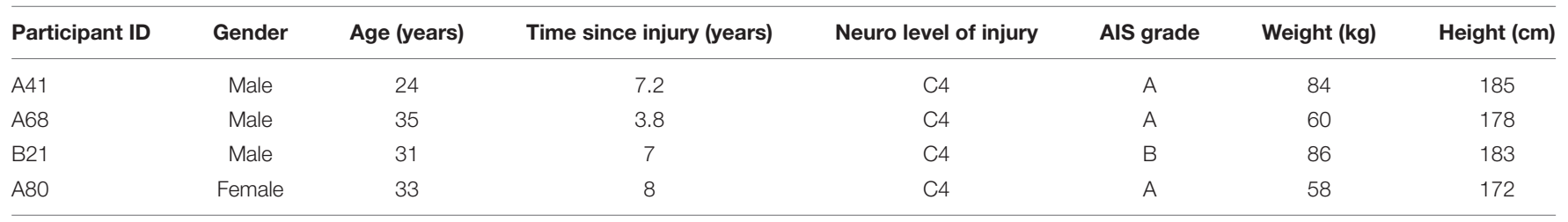

AIS, American spinal injury association Impairment Scale. 


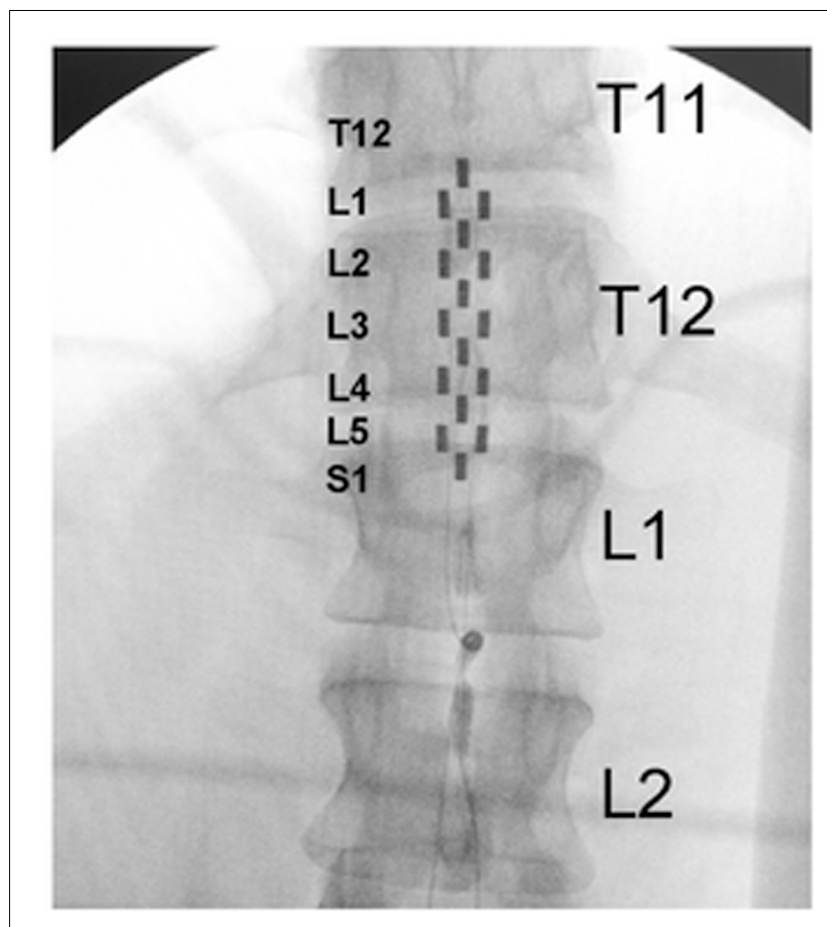

FIGURE 1 | Fluoroscopy showing electrode array location relative to thoracic and lumbar vertebrae. Large letters T11, T12, L1, and $L 2$ identify thoracic and lumbar vertebrae; small letters L1-S1 identify the spinal cord levels estimated by mapping with motor-evoked potentials.

voltage, frequency, and pulse width) were identified to maintain systolic blood pressure within the range of $110-120 \mathrm{mmHg}$ for research participants A41, A68, and B21 and 105-115 mmHg for research participant A80, without skeletal muscle activity of the lower extremities as assessed by electromyography in mapping experiments (Figure 2). This usually took two to three sessions that were limited to $2 \mathrm{~h}$. Then five 2 -h stimulation sessions occurred where the CV-scES stimulation voltage and frequency would have been adjusted as needed throughout the session in order to target systolic blood pressure within the 110-120 mmHg range (for participants $\mathrm{A} 41, \mathrm{~A} 68$, and $\mathrm{B} 21$ ) and $105-115 \mathrm{mmHg}$ range (for participant $\mathrm{A} 80$ ). Pulse width was $450 \mu \mathrm{s}$ in all sessions for all participants. Diastolic blood pressure and heart rate were also monitored to assure that they were maintained within normative ranges. Resting data were obtained for $15 \mathrm{~min}$ before and after the 2-h stimulation period. Data from the first five cardiovascular sessions obtained within 2 weeks are presented.

\section{Cardiovascular Data Acquisition and Analysis}

Noninvasive continuous blood pressure measured from a finger cuff by plethysmographic technique (Finometer Pro, FMS, Amsterdam, Netherlands) was calibrated (Schutte et al., 2003) and recorded continuously in cardiovascular sessions. The continuous data were sampled with $1000 \mathrm{~Hz}$ sampling rate with an $\mathrm{A} / \mathrm{D}$ converter device and computer interface (PowerLab 30/35 Series and LabChart, AD Instruments, Colorado Springs,
$\mathrm{CO}$, United States) and stored for offline analysis and were used to guide selection of initial stimulation parameters. Brachial blood pressure measured by oscillometric technique (Carescape V100, GE Healthcare, Milwaukee, WI, United States) was obtained every 2-4 min during the session to calibrate finger blood pressure measurements during the experiments and post-analyses. During offline analysis, beat-to-beat systolic and diastolic blood pressure were calculated as the peaks and nadirs of the finger blood pressure waveform, after finger blood pressure was calibrated to brachial levels and internal calibration artifacts were removed. Mean arterial pressure was calculated as one-third of systolic blood pressure plus two-third of diastolic blood pressure. Heart rate was calculated from the interval between two beats. Beat-to-beat pressures and heart rate were then averaged for every $1 \mathrm{~min}$ for statistical analysis. All analyses were performed with customized software in MATLAB (MathWorks, Natick, MA, United States).

\section{Statistics}

Data were summarized graphically using box plots. The box represents the interquartile range values $(25 \mathrm{th}, 50 \mathrm{th}$, and 75 th percentiles) and the whiskers extend to non-outliers minimum and maximum data points. Data points $1.5 \times$ IQR above the 75 th percentile or below the 25 th percentile were considered outliers. For each research participant and for the first session, we performed three comparisons for mean arterial pressure, systolic blood pressure, diastolic blood pressure, and heart rate: CV-scES (during stimulation) vs. pre-CV-scES (before stimulation); postCV-scES (after stimulation) vs. CV-scES (during stimulation); and post-CV-scES (after stimulation) vs. pre-CV-scES (before stimulation). To perform these comparisons, we used linear mixed models on logged values including the experimental phase (pre-CV-scES, CV-scES, and post-CV-scES) as fixed effect and the minutes as random effect nested within period phase, adjusted for the longitudinal nature of the data and the autoregressive nature of the data. Let $y$ represent the outcome (mean arterial pressure, systolic or diastolic blood pressure, or heart rate), the equation of the model was specified as follows: $\log \left(y_{i j}\right)=\beta_{0}+\beta_{1} *$ time $_{i j}+\beta_{2}$ phase $_{j}+\varepsilon_{i j}$ with $\varepsilon_{i j}=$ $\alpha_{0}+\alpha_{1} * \varepsilon_{i-1, j}$, where $i$ represents the time and $j$ represents the experimental phase rank (pre-CV-scES = phase $1, \mathrm{CV}$ scES = phase 2 , and post-CV-scES = phase 3 ). The three two-bytwo comparisons were obtained by constructing linear contrasts from the model.

Each delta mean arterial pressure, delta systolic blood pressure, and delta heart rate value during CV-scES were calculated as the difference of that value and the average of all pre-CV-scES values. These data were not normally distributed and they were also autoregressive ( $p$-value of serial correlation $<0.05)$. To test whether delta mean arterial pressure, delta systolic blood pressure, delta diastolic blood pressure, and delta heart rate are different from 0 , we hence used the signed rank test (comparing medians) adjusted for serial correlation using the method of effective sample size (Mitchell et al., 1966; Zwiers and Storch, 1995). Statistical analyses were performed in SAS 9.4 (SAS Institute, Inc., Cary, NC, United States). 
A

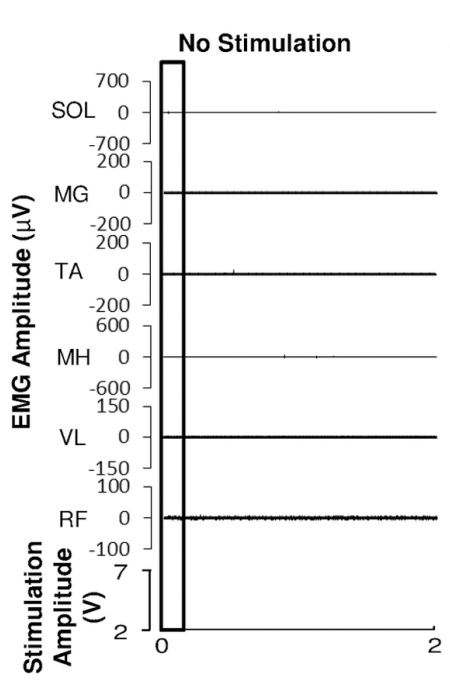

B

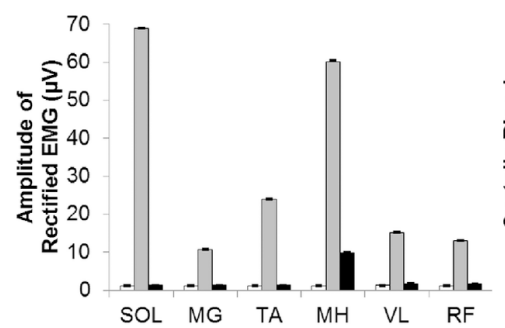

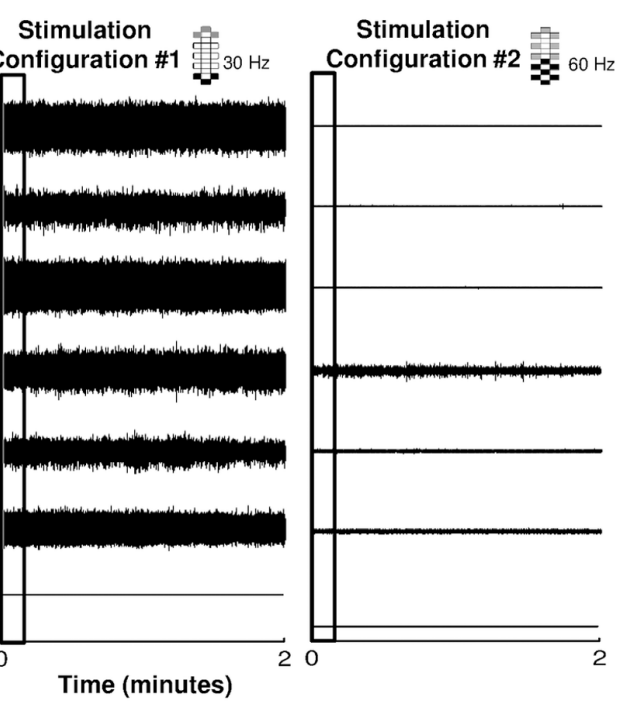
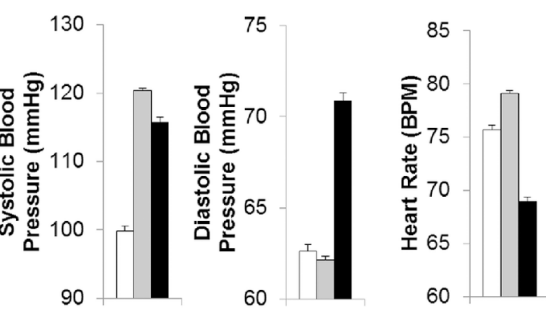

C
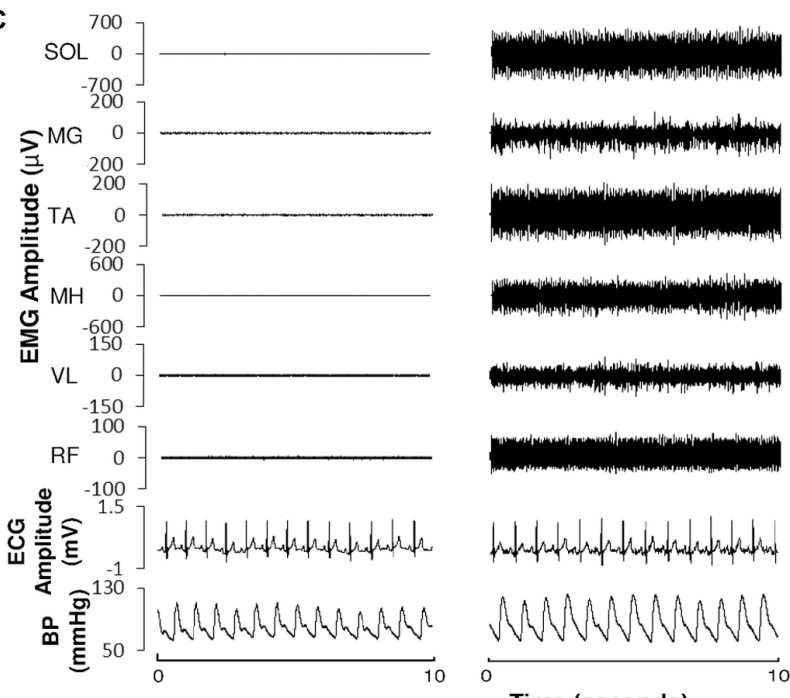

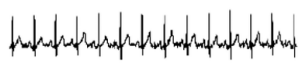

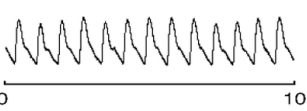

Time (seconds)

FIGURE 2 | Electromyography, electrocardiography, and blood pressure during different epidural stimulation configurations exemplified in one research participant (B21). (A) Electromyography (EMG) of the soleurs (SOL), medial gastrocnemius (MG), tibialis anterior (TA), medial hamstrings (MH), vastus lateralis (VL), and rectus femoris (RF) during 2 min of no stimulation (left), using CV-scES, $30 \mathrm{~Hz}$, Configuration No. 1 (middle) and using CV-scES, $60 \mathrm{~Hz}$, configuration No. 2 (right) in one research participant B21. The electrode selections and stimulation frequency were indicated on the top right of each panel. Gray boxes are cathodes and black boxes are anodes, white boxes are inactive electrodes. (B) Amplitude of rectified mean EMG, systolic blood pressure, diastolic blood pressure, and heart rate averaged over 2 min of no stimulation (white bars), configuration No. 1 (gray bars) and configuration No. 2 (black bars) from the data shown in (A). Error bars represent standard error. (C) EMG, ECG, and continuous blood pressure in the 10-s windows depicted by the black boxes in (A). 


\section{Determination of Initial CV-scES for Each Individual}

Each individual completes 3 days of motor mapping of the electrode encompassing voltage response and frequency response curves of local two anode-cathode combinations rostral caudal and left right.

To determine the initial CV-scES configurations, we record EMG activity to identify those that modulate blood pressure but do not elicit motor activity (Figure 2). We collected EMG at 2,000 $\mathrm{Hz}$ using a 24-channel hard-wired $\mathrm{AD}$ board and custom-written acquisition software (Labview, National Instruments, Austin, TX, United States). EMG (MotionLab Systems, Baton Rouge, LA, United States) from the soleus, medial gastrocnemius, tibialis anterior, medial hamstrings, rectus femoris, and vastus lateralis using bipolar surface electrodes with fixed inter electrode distance (Harkema et al., 1997). In addition, two surface electrodes were placed over the paraspinal muscles, symmetrically lateral to the epidural electrode array incision site. These two electrodes were used to record the stimulation artifact from the implanted electrode. EMG data were rectified and high-pass filtered at $40 \mathrm{~Hz}$ using Labview software customized by our laboratory. In these experiments we are very often modulating the parameters (anode, cathode selection; frequency and amplitude, number of programs) and the focus is to winnow down to successful CV-scES configurations. The goal is to find a $\mathrm{CV}$-scES configuration that can maintain a relatively stable blood pressure within non-injured defined normal ranges for $2 \mathrm{~h}$ without eliciting motor activity.

\section{RESULTS}

\section{Blood Pressure and Heart Rate Responses to CV-scES}

Cardiovascular parameters were normalized consistently in four individuals with chronic cervical SCI using participant-specific CV-scES. Prior to stimulation there is often variability in the systolic blood pressure especially when below $90 \mathrm{mmHg}$ as exemplified in participant A68 (Figure 3). This typically occurs is response to yawning or involuntary muscle activation. Systolic blood pressure was gradually increased to the $110-120$ or $105-115 \mathrm{mmHg}$ range using the participant-specific CV-scES configuration and could be maintained with minimal modulations in the stimulation amplitude. The stimulation amplitude was increased to increase systolic blood pressure and decreased to reduce systolic blood pressure to maintain the systolic blood pressure within the target range for $60 \mathrm{~min}$. Generally, heart rate was inversely related to systolic blood pressure with some variability over time.

For each research participant there were statistically significant increases in mean arterial pressure in response to $\mathrm{CV}$-scES (Figure 4A). The mean arterial pressure changes represented concomitant increases in both systolic and diastolic blood pressures as detailed in Table 2. This rise in blood pressure returned near or below each participant's baseline values within $15 \mathrm{~min}$ of $\mathrm{CV}$-scES cessation. Three individuals had no significant change in heart rate while A41 had a significant decrease during stimulation. After the CV-scES was turned off heart rate was significantly greater than the baseline for each participant (Figure 4B). The average increases in mean arterial pressure during CV-scES for all four participants were reproducible as shown from five sessions over a 2 -week period with similar responses among the participants (Figure 5 and Table 3). Individuals reported physical changes during these sessions including: (1) a feeling of alertness or heightened awareness; (2) increased ability to project their voice and carry on conversations; (3) increased capacity to breathe and cough; and (4) overall improved sense of well-being.

\section{Individual-Specific CV-scES Configurations}

Each individual required a specific and unique CV-scES configuration (anode-cathode electrode selection, pulse width, frequency, and amplitude) to consistently maintain normalized cardiovascular parameters (Figure 6). All four individuals had different configurations that maintained their systolic blood pressure stable within normal limits (Figures 6A-D). Also, those configurations were specific and the anode-cathode selection as well as the frequency interaction is important in enabling the consistent control of blood pressure as shown when comparing configurations that were not successful even when continually modulating stimulation amplitude (Figures 6E-H).

\section{DISCUSSION}

Persistent hypotension was resolved in four individuals with chronic cervical SCI within normal blood pressure ranges using lumbosacral scES with customized targeted configurations for cardiovascular function (CV-scES). In all four individuals there were significant and reproducible increases in systolic and diastolic blood pressures (Figures 3, 4 and Table 2). Heart rates decreased in one individual and remained relatively stable in the other three during stimulation. These individuals reported that they were invigorated, had better voice projection, were able to communicate more effectively, and could breathe and cough better during these stimulation periods. The normotensive pressures were maintained for $2 \mathrm{~h}$ with minor adjustments in amplitude or frequency and without activation of skeletal muscles. This effect was reproducible over multiple sessions (Figure 5), and returned to low blood pressures when the stimulation ceased. Our study shows that stimulating dorsal lumbosacral spinal cord can effectively and safely activate mechanisms to elevate blood pressures to a normal range from a chronic hypotensive state in humans with severe SCI with individual-specific CV-scES for a limited time period when the stimulation is present (Figure 6).

The eventual activation of sympathetic vasomotor efferents in the lumbosacral spinal cord causing vasoconstriction of the peripheral arteries leading to increase in venous return is a possible mechanism of the increase in blood pressure with $\mathrm{CV}$-scES. The rise in blood pressure then likely stimulated the SCI participants' intact baroreceptors in the aortic arch and carotid 


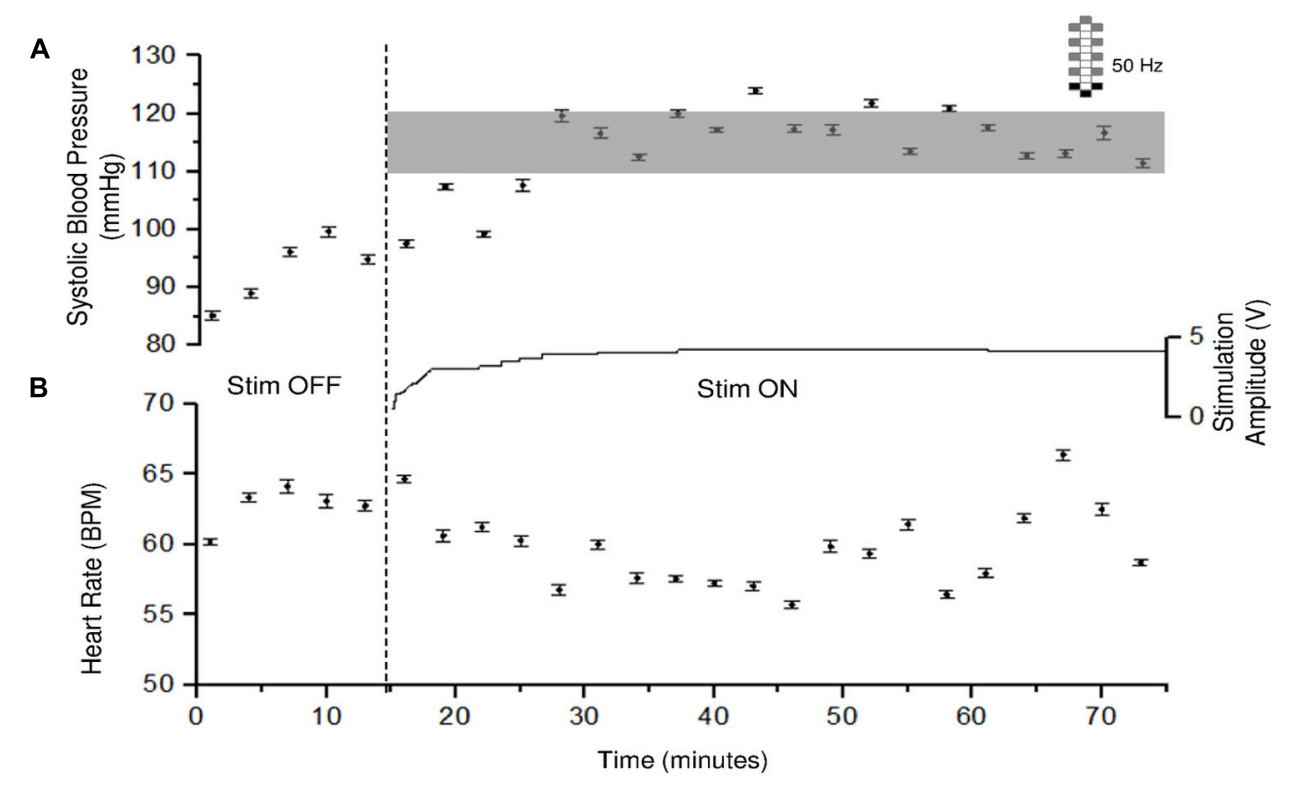

FIGURE 3 | Systolic blood pressure and heart rate responses to CV-scES in one research participant (A68). Systolic blood pressure gradually increased to target range and was maintained during 60 min of activation of stimulation (Stim ON) with minimal adjustment in stimulation amplitude, compared with baseline without stimulation (Stim OFF). Heart rate generally showed an inverse relationship with systolic blood pressure. Solid circles represent sitting systolic blood pressure (mmHg, top panel) and heart rate (beats per minute, bottom panel) averaged over every 3 min of beat-to-beat values (mean \pm SE). Gray shading area represents the target range of systolic blood pressure (110-120 $\mathrm{mmHg}$ ). Stimulation (Stim) amplitude is shown in the middle and was continuous throughout the 60 min. Frequency was constant at $50 \mathrm{~Hz}$. The vertical dash line indicates the start of stimulation. Electrode configuration is represented on the top right; gray boxes are cathodes, black boxes are anodes, and white boxes are inactive electrodes.

sinus decreasing the heart rate via increased parasympathetic tone maintaining a stabilized system. Yanagiya et al. (2004) provided evidence for controlling both pressor and depressor responses using ES in a model of a "bionic baroreflex" in the epidural space from T12 to L1 in six baroreflex-deafferentiated cats. This study attenuated orthostatic responses using a blood pressure monitor via an indwelling sensor to trigger appropriate scES in response to hypotension. Initially, head-up tilting of these cats led to a decrease in arterial pressure by $59 \mathrm{mmHg}$ in the first $30 \mathrm{~s}$. With use of scES these orthostatic responses significantly attenuated to $8 \mathrm{mmHg}$ at $30 \mathrm{~s}$.

For orthostatic hypotension or resting hypotension, the lower thoracic levels for scES may also be efficacious in raising blood pressure due to the effects of efferent stimulation on the splanchnic vascular bed via sympathetic vasomotor efferents (Hainsworth and Karim, 1976; Minson et al., 1999). Sato et al. (2002) demonstrated similar results in a rat model using an indwelling arterial pressure manometer triggering appropriate electrical stimulation of the vasomotor sympathetic nerve at celiac ganglion in response to hypotension after head-up tilting in 10 rats. Initially, head-up tilting led to a drop in arterial pressure of $52 \mathrm{mmHg}$ at $10 \mathrm{~s}$ which was decreased to $15 \mathrm{mmHg}$ after repeat head-up tilting using electrical stimulation. These attenuated hypotensive responses with electrical stimulation were similar to the control experiments with intact native baroreflex function. It is conceivable that splanchnic vasoconstriction also could have contributed to venous return and increased blood pressure in response to $\mathrm{CV}$-scES in our individuals with hypotension secondary to chronic SCI. However, it seems that the site of stimulation is more consistent with the sympathetic fibers described in the Yanagiya et al. (2004) study that the activation of the vasomotor sympathetic efferents from the lumbar cord may be the more prominent mechanism responsible for the effect observed in this study given the similar location and type of response. However, other mechanisms must also be considered, for example, given the dorsal location of the electrode, dorsal fibers that project to intermediolateral columns may reach and influence other levels of the spinal cord, and aspects of the autonomic regulatory system.

A proof of concept human scES trial for the clinical artificial bionic baroreflex system was tested in 21 patients undergoing total knee arthroplasty (Yamasaki et al., 2006). Lower thoracic level scES was effective in attenuating the hypotensive response after rapid onset of depression of blood pressure induced by sudden deflation of thigh tourniquet-simulated central baroreflex failure. The study used percutaneous electrodes with an artificially induced perturbation in blood pressure to provide evidence that the mechanisms described in their animal experiments (Sato et al., 2002; Yanagiya et al., 2004) were translatable to a clinical model. We now demonstrate that targeted and sustained CV-scES in the SCI population of the lumbosacral spinal cord has the potential for providing a similar therapeutic effect specifically in individuals with traumatic SCI.

Other human scES trials for the cardiovascular conditions of heart failure and hypertension had varied responses depending on their pathophysiological state and stimulation site and 


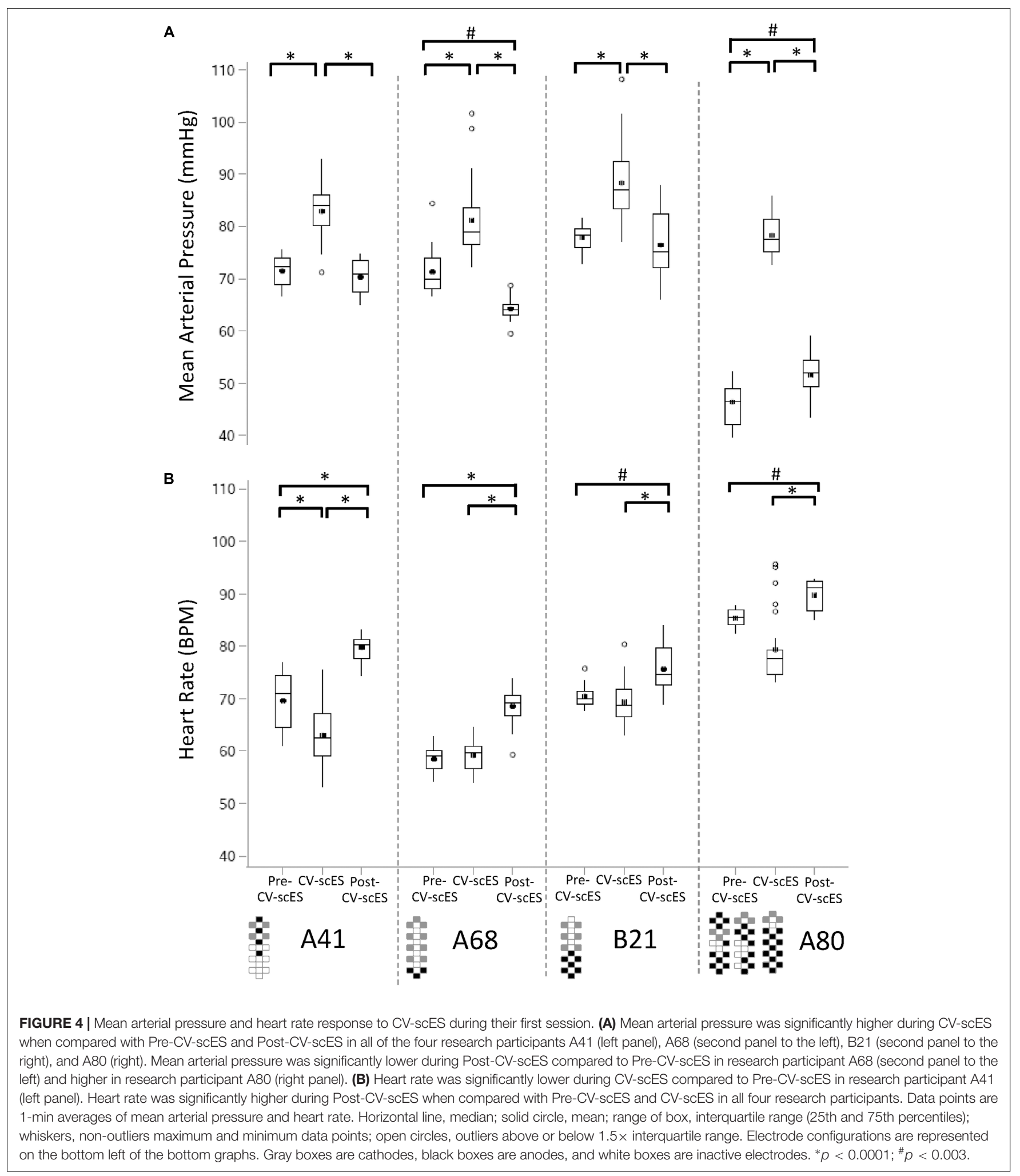

stimulation configuration (Schultz et al., 2007, 2011; Tse et al., 2015; Zipes et al., 2016). In the case of experimentally induced hypertension using a cold pressor test in individuals with an intact spinal cord who were normotensive, stimulation at higher levels (T1-T2 or T5-T6) had mixed results in attenuating the cardiovascular response (Schultz et al., 2007). A later study in normotensive and hypertensive individuals also with an intact spinal cord did not observe significant changes in mean arterial 
TABLE 2 | Sitting systolic and diastolic blood pressure before and during CV-scES at the first session day of each participant.

\begin{tabular}{|c|c|c|c|c|c|c|}
\hline \multirow[b]{2}{*}{ Participant ID } & \multicolumn{3}{|c|}{ Systolic blood pressure (mmHg) } & \multicolumn{3}{|c|}{ Diastolic blood pressure $(\mathrm{mmHg})$} \\
\hline & Pre-CV-scES & CV-scES & $p$-value & Pre-CV-scES & CV-scES & $p$-value \\
\hline A41 & $101 \pm 4$ & $111 \pm 5$ & $<0.0001$ & $57 \pm 2$ & $69 \pm 5$ & $<0.0001$ \\
\hline A68 & $97 \pm 7$ & $112 \pm 11$ & 0.0001 & $59 \pm 4$ & $66 \pm 5$ & $<0.0001$ \\
\hline $\mathrm{B} 21$ & $106 \pm 5$ & $118 \pm 9$ & $<0.0001$ & $64 \pm 2$ & $74 \pm 6$ & 0.0002 \\
\hline A80 & $65 \pm 5$ & $109 \pm 8$ & $<0.0001$ & $37 \pm 3$ & $63 \pm 3$ & $<0.0001$ \\
\hline
\end{tabular}

Data presented as mean $\pm S D$ of minute-by-minute time series. p-value, CV-scES vs. Pre-CV-scES.
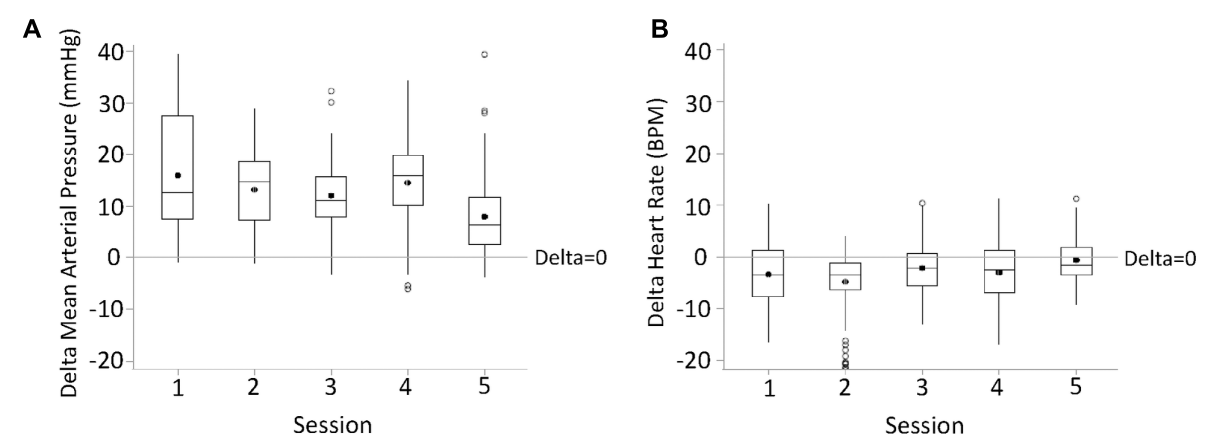

FIGURE 5 | Changes in mean arterial pressure (delta mean arterial pressure, A) and heart rate (delta heart rate, B) during the first half an hour of active CV-scES from Pre-CV-scES, combined for four research participants averaged for five sessions. Mean arterial pressure increased, and heart rate decreased, from Pre-CV-scES to CV-scES in all five sessions. Data points are 1-min averages of mean arterial pressure and heart rate subtracted from mean values of Pre-CV-scES. Horizontal line, median; solid circle, mean; range of box, interquartile range (25th and 75th percentiles); whiskers, non-outliers maximum and minimum data points; open circles, outliers above or below $1.5 \times$ interquartile range. Delta of mean arterial pressure and heart rate of all sessions were significantly different from zero $(p<0.05)$.

pressure with epidural stimulation at T5-T6 although they reported some trends and effects on heart rate variability (Schultz et al., 2007). The location and parameters of stimulation were targeted based on animal studies to induce vasodialation (Croom et al., 1997; Tanaka et al., 2001, 2004). The investigators did observe modulation of blood pressure albeit with variability. This may be attributed to individuals with intact spinal cord and cardiovascular regulatory systems can respond and compensate for the stimulatory effects. This may further be supported by the observation that the hypertensive group had more modulation by the stimulation than the normotensive group. These studies differ from our observations in the location of stimulation, the limited parameters of stimulation they were able to assess, and their targeted mechanism of vasodialation. In our study with the permanent implant, we were able to take several hours to identify specific configurations that increased blood pressure that was sub-motor threshold. These studies demonstrated that scES proved to be safe, positioning of the electrode and parameter selection influenced the effect, and the spinal cord stimulation may have clinical implications for hypertension.

Another approach of epidural stimulation in the high thoracic region (T1-T3) in a small cohort human study (Tse et al., 2015) stimulated to improve left ventricular myocardial contractile function and ejection fraction without increasing oxygen consumption based on earlier human studies (Liu et al., 2012). This study showed safety and early feasiblily although a follow-up randomized study of 66 patients did not show a significant difference from a comparison group of a cardioverter-defibrillator group with left ventricular ejection fraction as the primary outcome measure (Zipes et al., 2016). Interestingly to note in the larger cohort is that the study was stopped prior to the planned enrollment of 195 subjects and statistical significance between the two groups was not sufficiently powered to see a significant difference between the two groups. Also, spinal cord stimulation was arbritrarily chosen from animal studies and on the basis of those used for patients with intractable angina. Physiological surrogates were not used for each individual to identify stimulation parameters. We found that to maintain normative blood pressures each person required unique CV-scES parameters and it took mapping for several hours using physiological measures to identify

TABLE 3 | Change in systolic and diastolic blood pressure during CV-scES compared with baseline without CV-scES, combining four participants for each of the five session days.

\begin{tabular}{lcccc}
\hline $\begin{array}{c}\text { Session } \\
\text { number }\end{array}$ & $\begin{array}{c}\text { Change in } \\
\text { systolic blood } \\
\text { pressure }(\mathbf{m m H g})\end{array}$ & $\boldsymbol{p}$-value & $\begin{array}{c}\text { Change in } \\
\text { diastolic blood } \\
\text { pressure (mmHg) }\end{array}$ & p-value \\
\hline 1 & $20 \pm 16$ & 0.0418 & $14 \pm 9$ & 0.0221 \\
2 & $18 \pm 12$ & 0.0184 & $11 \pm 6$ & 0.0026 \\
3 & $14 \pm 14$ & 0.0004 & $10 \pm 8$ & $<0.0001$ \\
4 & $17 \pm 10$ & 0.0043 & $13 \pm 7$ & 0.0221 \\
\hline
\end{tabular}

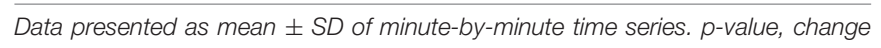
in systolic or diastolic blood pressure vs. $0 \mathrm{mmHg}$. 


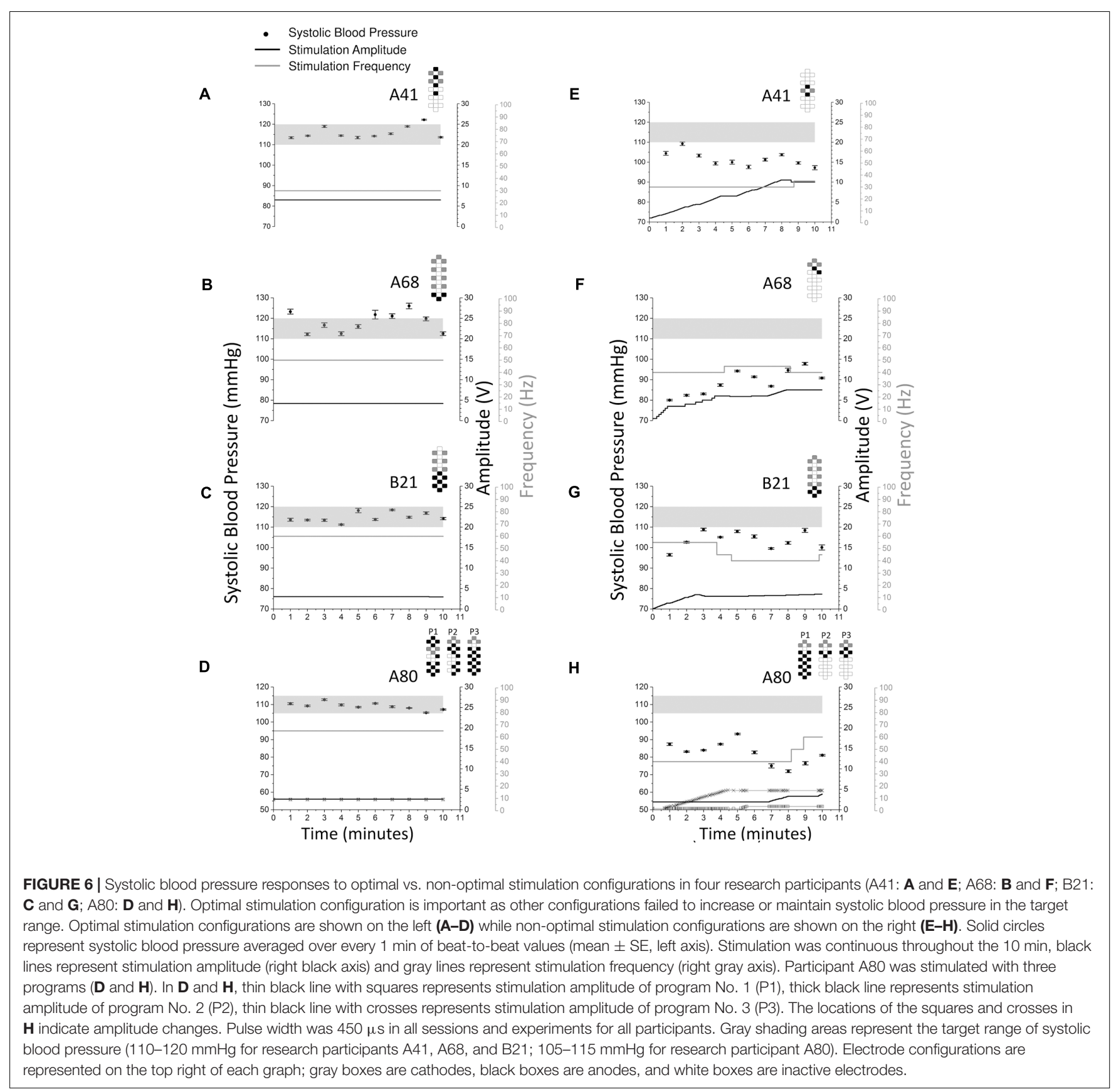

them. These studies concluded that the spinal cord stimulation was safe; however, there is significant variability with the site of stimulation, the pathophysiology, as the configuration selection all clearly will impact the outcome and need to be carefully considered when designing clinical studies and treatments.

The current study, when also taken in context with other recent studies of epidural stimulation, demonstrates that individuals diagnosed with motor complete SCI may have significant potential for recovery. In the presence of epidural stimulation of spinal networks there now is evidence that individuals who have been diagnosed with motor complete SCI can regain volitional movement (Angeli et al., 2014) and the ability to stand (Rejc et al., 2015, 2017), and in this study we show that they can potentially resolve another devastating consequence of SCI, cardiovascular dysfunction. These reversals of the repercussions of SCI in those with the most severe injuries, those determined that there is the most isolation from supraspinal input, provide us with the ability to gain insights to the infrastructure and capacity for plasticity of the human spinal circuitry (Grillner and Wallen, 2004). A key factor in these studies is that each motor behavior and physiological response has its own unique stimulation configuration indicating that the successful execution of the response is dependent on the 
appropriate excitability of the spinal network. Each individual also has a unique configuration and that may have many contributing factors including placement of electrode, differences among injuries, time since injury, pharmaceuticals, and levels of activity.

Epidural stimulation directly activated the spinal circuitry and immediately improved blood pressure in these individuals with SCI. There are other non-invasive modalities that can access the spinal circuitry such as transcutaneous stimulation (Gerasimenko et al., 2015, 2016). Locomotor training is an activity-based therapy that activates the neuromuscular system below the level of injury (Harkema et al., 1997; Beres-Jones and Harkema, 2004) and has been shown to improve blood pressure in individuals with incomplete SCI (Ditor et al., 2005). Both of these target the spinal circuitry that is available after SCI and also is a significant target for therapy (Harkema et al., 2012a,b). These findings provide a proof of principle that there may be many avenues available for significant recovery for individuals who live with paralysis that have not yet been fully taken advantage of in clinical environments.

The availability of implantable scES devices provides a novel treatment for chronic SCI patients to improve symptomatic resting and orthostatic hypotension. Longer term studies are needed to assess cardiovascular effects of CV-scES on myocardial structure and function, vascular biology, and clinical outcomes in this population. Development of vascular pressure sensing devices that can activate appropriate CV-scES as a closed feedback system is essential for home and community use and could ameliorate this major impairment to quality of life in the SCI population. These findings are important not only to the chronic SCI population with substantial morbidity and mortality related to excess cardiovascular disease including extreme instability and liability of blood pressure control, but also to other disease states with significant morbidity from orthostatic hypotension such as multiple system atrophy (previously called the Shy-Drager syndrome) or baroreceptor-deafferentiated states such as those that occurs after carotid sinus disruption from trauma, vascular surgery, or tumor involvement. Other dietary treatments such as salt loading or pharmacologic therapy with adrenergic agonists do not consistently prevent orthostatic hypotension and can lead to supine hypertension. CV-scES provides a potential unique treatment option for SCI patients to normalize blood pressure and alleviates the adverse impacts of chronic low blood pressure and orthostatic hypotension on physical, emotional, and social well-being (Carlozzi et al., 2013).

\section{LIMITATIONS AND FUTURE STUDIES}

This study was limited to four individuals and needs to be repeated in a larger number of people with chronic SCI over

\section{REFERENCES}

Angeli, C. A., Edgerton, V. R., Gerasimenko, Y. P., and Harkema, S. J. (2014). Altering spinal cord excitability enables voluntary movements after chronic longer periods of time to understand the broader application and to continue to understand the human spinal circuitry and the capacity of recovery after SCI. Further analyses of mechanisms focused on heart rate and blood pressure variability and poststimulation effects are needed. Larger, prolonged studies of this potential approach as a therapy are warranted to assess additional cardiovascular effects and whether it can improve symptoms or outcomes in other diseases characterized by low blood pressure and orthostatic hypotension. Closed-looped control systems need to be developed to allow monitoring and usability in real-life environments and in varied situations that will induce challenges to the cardiovascular system. Long-term effects of scES on autonomic dysreflexia and the respiratory system both related to these cardiovascular deficits also need to be studied.

\section{AUTHOR CONTRIBUTIONS}

$\mathrm{SH}$ and $\mathrm{CA}$ designed the study. $\mathrm{GH}$ and $\mathrm{MB}$ provided the medical oversight. MB performed the implant procedure with $\mathrm{SH}, \mathrm{CA}$, and $\mathrm{YC}$, providing the physiological data during the surgical procedure. SH identified the configurations for the cardiovascular stimulation, provided oversight for data acquisition and analyses, and performed cardiovascular sessions. SW and BU performed the data acquisition and analyses. All authors were involved in data interpretation and manuscript preparation.

\section{FUNDING}

This study was supported by the Craig H. Neilsen Foundation, The Leona M. and Harry B. Helmsley Charitable Trust, University of Louisville Hospital, Christopher \& Dana Reeve Foundation, and Medtronic Plc.

\section{ACKNOWLEDGMENTS}

We are indebted to our research participants for their courage, dedication, motivation, and perseverance that made these research findings possible. We would like to express our appreciation to Richard Seither, Brittany Castaneda, Katelyn Smith, and Sean Stills for their invaluable support of the research participants; Dr. Darryl Kaelin and Yukishia Austin, for medical oversight; J. Erin Wyles, Lisa Potts, and Christie Ferreira for data acquisition and analysis; Dengzhi Wang and Hanna Martin for data analysis; and our entire research staff for administrative, engineering, and additional research support.

complete paralysis in humans. Brain 137(Pt 5), 1394-1409. doi: 10.1093/brain/ awu038

Aslan, S. C., Randall, D. C., Krassioukov, A. V., Phillips, A., and Ovechkin, A. V. (2016). Respiratory training improves blood pressure regulation in individuals 
with chronic spinal cord injury. Arch. Phys. Med. Rehabil. 97, 964-973. doi: 10.1016/j.apmr.2015.11.018

Beres-Jones, J. A., and Harkema, S. J. (2004). The human spinal cord interprets velocity-dependent afferent input during stepping. Brain 127(Pt 10), 2232-2246. doi: 10.1093/brain/awh252

Carlozzi, N. E., Fyffe, D., Morin, K. G., Byrne, R., Tulsky, D. S., Victorson, D., et al. (2013). Impact of blood pressure dysregulation on health-related quality of life in persons with spinal cord injury: development of a conceptual model. Arch. Phys. Med. Rehabil. 94, 1721-1730. doi: 10.1016/j.apmr.2013.02.024

Chao, C. Y., and Cheing, G. L. (2005). The effects of lower-extremity functional electric stimulation on the orthostatic responses of people with tetraplegia. Arch. Phys. Med. Rehabil. 86, 1427-1433. doi: 10.1016/j.apmr.2004.12.033

Croom, J. E., Foreman, R. D., Chandler, M. J., and Barron, K. W. (1997). Cutaneous vasodilation during dorsal column stimulation is mediated by dorsal roots and CGRP. Am. J. Physiol. 272(2 Pt 2), H950-H957. doi: 10.1152/ajpheart.1997.272. 2.H950

Ditor, D. S., Macdonald, M. J., Kamath, M. V., Bugaresti, J., Adams, M., McCartney, N., et al. (2005). The effects of body-weight supported treadmill training on cardiovascular regulation in individuals with motor-complete SCI. Spinal Cord 43, 664-673. doi: 10.1038/sj.sc.3101785

Freeman, R. (2008). Current pharmacologic treatment for orthostatic hypotension. Clin. Auton. Res. 18(Suppl. 1), 14-18. doi: 10.1007/s10286-007-1003-1

Gerasimenko, Y., Gad, P., Sayenko, D., McKinney, Z., Gorodnichev, R., Puhov, A., et al. (2016). Integration of sensory, spinal, and volitional descending inputs in regulation of human locomotion. J. Neurophysiol. 116, 98-105. doi: 10.1152/jn. 00146.2016

Gerasimenko, Y. P., Lu, D. C., Modaber, M., Zdunowski, S., Gad, P., Sayenko, D. G., et al. (2015). Noninvasive reactivation of motor descending control after paralysis. J. Neurotrauma 32, 1968-1980. doi: 10.1089/neu.2015.4008

Grillner, S., and Wallen, P. (2004). Innate versus learned movements-a false dichotomy? Prog. Brain Res. 143, 3-12.

Hainsworth, R., and Karim, F. (1976). Responses of abdominal vascular capacitance in the anaesthetized dog to changes in carotid sinus pressure. J. Physiol. 262, 659-677. doi: 10.1113/jphysiol.1976.sp011614

Harkema, S., Gerasimenko, Y., Hodes, J., Burdick, J., Angeli, C., Chen, Y., et al. (2011). Effect of epidural stimulation of the lumbosacral spinal cord on voluntary movement, standing, and assisted stepping after motor complete paraplegia: a case study. Lancet 377, 1938-1947. doi: 10.1016/S0140-6736(11) 60547-3

Harkema, S. J., Ferreira, C. K., van den Brand, R. J., and Krassioukov, A. V. (2008). Improvements in orthostatic instability with stand locomotor training in individuals with spinal cord injury. J. Neurotrauma 25, 1467-1475. doi: $10.1089 /$ neu.2008.0572

Harkema, S. J., Hillyer, J., Schmidt-Read, M., Ardolino, E., Sisto, S., and Behrman, A. (2012a). Locomotor training: as a treatment of spinal cord injury and in the progression of neurological rehabilitation. Arch. Phys. Med. Rehabil. 93, 1588-1597. doi: 10.1016/j.apmr.2012.04.032

Harkema, S. J., Hurley, S. L., Patel, U. K., Requejo, P. S., Dobkin, B. H., and Edgerton, V. R. (1997). Human lumbosacral spinal cord interprets loading during stepping. J. Neurophysiol. 77, 797-811. doi: 10.1152/jn.1997.77.2.797

Harkema, S. J., Schmidt-Read, M., Lorenz, D. J., Edgerton, V. R., and Behrman, A. L. (2012b). Balance and ambulation improvements in individuals with chronic incomplete spinal cord injury using locomotor training-based rehabilitation. Arch. Phys. Med. Rehabil. 93, 1508-1517. doi: 10.1016/j.apmr. 2011.01.024

Krassioukov, A., and Claydon, V. E. (2006). The clinical problems in cardiovascular control following spinal cord injury: an overview. Prog. Brain Res. 152, 223-229. doi: 10.1016/S0079-6123(05)52014-4

Krassioukov, A., Eng, J. J., Warburton, D. E., and Teasell, R. (2009). A systematic review of the management of orthostatic hypotension after spinal cord injury. Arch. Phys. Med. Rehabil. 90, 876-885. doi: 10.1016/j.apmr.2009. 01.009

Liu, Y., Yue, W. S., Liao, S. Y., Zhang, Y., Au, K. W., Shuto, C., et al. (2012). Thoracic spinal cord stimulation improves cardiac contractile function and myocardial oxygen consumption in a porcine model of ischemic heart failure. J. Cardiovasc. Electrophysiol. 23, 534-540. doi: 10.1111/j.1540-8167.2011. 02230.x
Mathias, C. J., and Kimber, J. R. (1998). Treatment of postural hypotension. J. Neurol. Neurosurg. Psychiatry 65, 285-289. doi: 10.1136/jnnp.65.3.285

Minson, C. T., Wladkowski, S. L., Pawelczyk, J. A., and Kenney, W. L. (1999). Age, splanchnic vasoconstriction, and heat stress during tilting. Am. J. Physiol. 276(1 Pt 2), R203-R212. doi: 10.1152/ajpregu.1999.276.1.R203

Mitchell, J. M., Dzerdzeevskii, B., Flohn, H., Hofmeyr, W. L., Lamb, H. H., Rao, K. N., et al. (1966). Technical note No. 79. climatic change. (Report of a working group of the commission for climatology). World Meteorol. Organ. 195:179.

Rejc, E., Angeli, C., and Harkema, S. (2015). Effects of lumbosacral spinal cord epidural stimulation for standing after chronic complete paralysis in humans. PLoS One 10:e0133998. doi: 10.1371/journal.pone.0133998

Rejc, E., Angeli, C. A., Bryant, N., and Harkema, S. J. (2017). Effects of stand and step training with epidural stimulation on motor function for standing in chronic complete paraplegics. J. Neurotrauma 34, 1787-1802. doi: 10.1089/neu. 2016.4516

Sato, T., Kawada, T., Sugimachi, M., and Sunagawa, K. (2002). Bionic technology revitalizes native baroreflex function in rats with baroreflex failure. Circulation 106, 730-734. doi: 10.1161/01.CIR.0000024101.77521.4D

Schultz, D. M., Musley, S., Beltrand, P., Christensen, J., Euler, D., and Warman, E. (2007). Acute cardiovascular effects of epidural spinal cord stimulation. Pain Physician 10, 677-685.

Schultz, D. M., Zhou, X., Singal, A., and Musley, S. (2011). Cardiovascular effects of spinal cord stimulation in hypertensive patients. Pain Physician 14, 1-14.

Schutte, A. E., Huisman, H. W., Van Rooyen, J. M., Oosthuizen, W., and Jerling, J. C. (2003). Sensitivity of the Finometer device in detecting acute and mediumterm changes in cardiovascular function. Blood Press. Monit. 8, 195-201. doi: 10.1097/01.mbp.0000103280.95050.f7

Tanaka, S., Barron, K. W., Chandler, M. J., Linderoth, B., and Foreman, R. D. (2001). Low intensity spinal cord stimulation may induce cutaneous vasodilation via CGRP release. Brain Res. 896, 183-187. doi: 10.1016/S00068993(01)02144-8

Tanaka, S., Komori, N., Barron, K. W., Chandler, M. J., Linderoth, B., and Foreman, R. D. (2004). Mechanisms of sustained cutaneous vasodilation induced by spinal cord stimulation. Auton. Neurosci. 114, 55-60. doi: 10.1016/j.autneu. 2004.07.004

Tse, H. F., Turner, S., Sanders, P., Okuyama, Y., Fujiu, K., Cheung, C. W., et al. (2015). Thoracic spinal cord stimulation for heart failure as a restorative treatment (SCS HEART study): first-in-man experience. Heart Rhythm 12, 588-595. doi: 10.1016/j.hrthm.2014.12.014

Wecht, J. M., and Bauman, W. A. (2017). Implication of altered autonomic control for orthostatic tolerance in SCI. Auton. Neurosci. 209, 51-58. doi: 10.1016/j. autneu.2017.04.004

Yamasaki, F., Ushida, T., Yokoyama, T., Ando, M., Yamashita, K., and Sato, T. (2006). Artificial baroreflex: clinical application of a bionic baroreflex system. Circulation 113, 634-639. doi: 10.1161/circulationaha.105.587915

Yanagiya, Y., Sato, T., Kawada, T., Inagaki, M., Tatewaki, T., Zheng, C., et al. (2004). Bionic epidural stimulation restores arterial pressure regulation during orthostasis. J Appl. Physiol. 97, 984-990. doi: 10.1152/japplphysiol.00162.2004

Zipes, D. P., Neuzil, P., Theres, H., Caraway, D., Mann, D. L., Mannheimer, C., et al. (2016). Determining the feasibility of spinal cord neuromodulation for the treatment of chronic systolic heart failure: the DEFEAT-HF study. JACC Heart Fail 4, 129-136. doi: 10.1016/j.jchf.2015.10.006

Zwiers, F. W., and Storch, H. V. (1995). Taking serial correlation into account in tests of the mean. J. Clim. 8, 336-351. doi: 10.1175/1520-0442(1995)008<0336: TSCIAI $>2.0 . \mathrm{CO} ; 2$

Conflict of Interest Statement: The authors declare that the research was conducted in the absence of any commercial or financial relationships that could be construed as a potential conflict of interest.

Copyright $\odot 2018$ Harkema, Wang, Angeli, Chen, Boakye, Ugiliweneza and Hirsch. This is an open-access article distributed under the terms of the Creative Commons Attribution License (CC BY). The use, distribution or reproduction in other forums is permitted, provided the original author(s) and the copyright owner are credited and that the original publication in this journal is cited, in accordance with accepted academic practice. No use, distribution or reproduction is permitted which does not comply with these terms. 